\title{
A QUINTUPLE LAW FOR MARKOV ADDITIVE PROCESSES WITH PHASE-TYPE JUMPS
}

\author{
LOTHAR BREUER, ${ }^{*}$ University of Kent
}

\begin{abstract}
We consider a Markov additive process (MAP) with phase-type jumps, starting at 0 . Given a positive level $u$, we determine the joint distribution of the undershoot and overshoot of the first jump over the level $u$, the maximal level before this jump, the time of attaining this maximum, and the time between the maximum and the jump. The analysis is based on first passage times and time reversion of MAPs. A marginal of the derived distribution is the Gerber-Shiu function, which is of interest to insurance risk. Several examples serve to compare the present result with the literature.
\end{abstract}

Keywords: Markov additive process; undershoot; surplus; ruin; Gerber-Shiu function

2010 Mathematics Subject Classification: Primary 60J25

Secondary 60G51; 91B30

\section{Introduction}

In recent literature on insurance risk, the undershoot of a variety of stochastic processes has been of considerable interest under the name of 'surplus prior to ruin'. Mostly, it has been investigated within the framework of the so-called Gerber-Shiu (discounted penalty) function, which describes the joint distribution of the time to ruin, the surplus prior to ruin, and the deficit at ruin. Some examples of stochastic processes for which results are available are the compound Poisson model [16], its perturbed [12], [15], [22] and Markov-modulated [4], [25] versions, the Lévy risk process [14], the fluid flow model [1], [8], the Sparre Andersen model with Erlang interclaim times [17] as well as its perturbed version [20]. This list is by no means exhaustive, but one can already see that all these models are special instances of Markov additive processes (MAPs). The almost universal approach in the literature is to derive some (defective) renewal equations, starting from a set of differential equations that can be obtained via Itô's formula or the infinitesimal generator of the surplus process (see the discussion to [21]).

From another perspective, a recent paper by Doney and Kyprianou [13] provides a formula for the joint distributions of the space-time positions of overshoots and undershoots for Lévy processes (see Theorem 3 therein, with Example 8 dedicated to insurance risk). Their approach of analysis is more along the classical lines of fluctuation theory, using the ladder height process and time reversal. For the case of Lévy processes with phase-type jumps, the ladder process is derived explicitly in [27]. Related results on MAPs can be found in [19].

The present paper aims to apply the classical approach of ladder heights and time reversal to the class of MAPs with phase-type jumps. Our main result is an explicit formula for the measure

$$
\mathrm{E}\left(\exp \left(-\gamma \tilde{G}_{\tilde{\tau}(u)}-\gamma^{*}\left(\tilde{\tau}(u)-\tilde{G}_{\tilde{\tau}(u)}\right)\right), \tilde{M}_{\tilde{\tau}(u)} \in \mathrm{d} z, U_{\tilde{\tau}(u)} \in \mathrm{d} x, O_{\tilde{\tau}(u)} \in \mathrm{d} y\right),
$$

Received 15 August 2008; revision received 2 March 2010.

* Postal address: Institute of Mathematics and Statistics, University of Kent, Canterbury CT2 7NF, UK.

Email address: 1.breuer@kent.ac.uk 
where $\tilde{X}=\left(\tilde{X}_{t}: t \geq 0\right)$ is the level process of a MAP, $\tilde{\tau}(u)$ is the first passage time over some level $u>0$, the $\gamma, \gamma * \geq 0$ are time discounting factors (which can be seen as the variables for the Laplace transforms of $\tilde{G}_{\tilde{\tau}(u)}$ and $\left.\tilde{\tau}(u)-\tilde{G}_{\tilde{\tau}(u)}\right), \tilde{M}_{\tilde{\tau}(u)}$ is the supremum of $\tilde{\mathcal{X}}$ before the passage time $\tilde{\tau}(u), \tilde{G}_{\tilde{\tau}(u)}$ is the time of attaining this supremum, and $U_{\tilde{\tau}(u)}:=u-\tilde{X}_{\tilde{\tau}(u)-}$ and $O_{\tilde{\tau}(u)}:=\tilde{X}_{\tilde{\tau}(u)}-u$ respectively denote the undershoot and overshoot at time $\tilde{\tau}(u)$. This result will provide all the information (and more) that is usually contained in the Gerber-Shiu function.

Of fundamental use in this paper will be the recent determination of the Laplace transform of first passage times for MAPs as given in [11]. A second pillar of the present work is Theorem 2.5 of [4] (see also [2, Theorem 3.1] for a queueing context), which yields a relation between an occupation measure and the ladder height via time reversal. The original result was presented in the framework of the Markov-modulated compound Poisson model.

In the following section we shall collect all the necessary preliminary results that we shall need later on. In particular, we simplify the results from [11] for the special kind of MAPs that we employ in this paper. Section 3 contains the main result with some corollaries. Finally, in Section 4 we present applications to insurance risk, in particular the Gerber-Shiu function, and compare the present result with existing results in the literature.

\section{Preliminaries}

\subsection{Markov additive processes with phase-type jumps}

Let $\tilde{\mathcal{g}}=\left(\tilde{J}_{t}: t \geq 0\right)$ be an irreducible Markov (jump) process with finite state space $\tilde{E}$ and infinitesimal generator matrix $\tilde{Q}=\left(\tilde{q}_{i j}\right)_{i, j \in \tilde{E}}$. We call $\tilde{J}_{t}$ the phase at time $t \geq 0$ (another common name is regime). Define the real-valued process $\tilde{X}=\left(\tilde{X}_{t}: t \geq 0\right)$ as evolving like a Lévy process $\tilde{X}^{(i)}$ with parameters $\tilde{\mu}_{i}$ (drift), $\tilde{\sigma}_{i}^{2}$ (variation), and $\tilde{v}_{i}$ (Lévy measure) during intervals when the phase equals $i \in \tilde{E}$. Whenever $\tilde{g}$ jumps from a state $i \in \tilde{E}$ to another state $j \in \tilde{E}, j \neq i$, this may be accompanied by a jump of $\tilde{X}$ with some distribution function $\tilde{F}_{i j}$. Then the two-dimensional process $(\tilde{\mathcal{X}}, \tilde{\mathcal{g}})$ is called a MAP. A MAP can also be defined by the following property (see $[5$, Section XI.2a]): $(\tilde{X}, \tilde{\mathcal{g}})$ is a Markov process such that

$$
\mathrm{E}\left(f\left(\tilde{X}_{t+s}-\tilde{X}_{t}\right) g\left(\tilde{J}_{t+s}\right) \mid \mathcal{F}_{t}, \tilde{J}_{t}=i\right)=\mathrm{E}\left(f\left(\tilde{X}_{s}\right) g\left(\tilde{J}_{s}\right) \mid \tilde{X}_{0}=0, \tilde{J}_{0}=i\right)
$$

holds for all $s, t>0$ and $i \in E$, where $f$ and $g$ are measurable functions and $\left(\mathcal{F}_{t}: t \geq 0\right)$ denotes the canonical filtration of $(\tilde{X}, \tilde{g})$. For a textbook introduction to MAPs, see [5, Chapter XI].

Denote the indicator function of a set $A$ by $1_{A}$. We assume that the Lévy measures $\tilde{v}_{i}$ have the form

$$
\tilde{v}_{i}(\mathrm{~d} x)=\lambda_{i}^{+} 1_{\{x>0\}} \alpha^{(i i)+} \exp \left(T^{(i i)+} x\right) \eta^{(i i)+} \mathrm{d} x+\lambda_{i}^{-} 1_{\{x<0\}} \alpha^{(i i)-} \exp \left(-T^{(i i)-} x\right) \eta^{(i i)-} \mathrm{d} x
$$

for all $i \in \tilde{E}$, where $\lambda_{i}^{ \pm} \geq 0$ and $\left(\alpha^{(i i) \pm}, T^{(i i) \pm}\right)$ are representations of phase-type distributions without an atom at 0 . The $\eta^{(i i) \pm}:=-T^{(i i) \pm} \mathbf{1}$ are called the exit vectors, where $\mathbf{1}$ denotes a column vector of appropriate dimension with all entries being 1 . This means that the jump process induced by the Lévy measure $\tilde{v}_{i}$ is compound Poisson with jump sizes of a doubly phase-type (PH) distribution. Denote the order of $\mathrm{PH}\left(\alpha^{(i i) \pm}, T^{(i i) \pm}\right)$ by $m_{i i}^{ \pm}$. Furthermore, write $\lambda_{i}:=\lambda_{i}^{+}+\lambda_{i}^{-}$.

Likewise, let $p_{i j}^{+}$and $p_{i j}^{-}$denote the probability that a positive or, respectively, negative jump is induced by a phase change from $i \in \tilde{E}$ to $j \in \tilde{E}$, and assume that these jumps have a $\mathrm{PH}\left(\alpha^{(i j) \pm}, T^{(i j) \pm}\right)$ distribution without an atom at 0 . Note that $p_{i j}^{+}+p_{i j}^{-} \leq 1$ for all $i, j \in \tilde{E}$. Let $m_{i j}^{ \pm}$denote the order of $\mathrm{PH}\left(\alpha^{(i j) \pm}, T^{(i j) \pm}\right)$ and define $\eta^{(i j) \pm}:=-T^{(i j) \pm} \mathbf{1}$. 


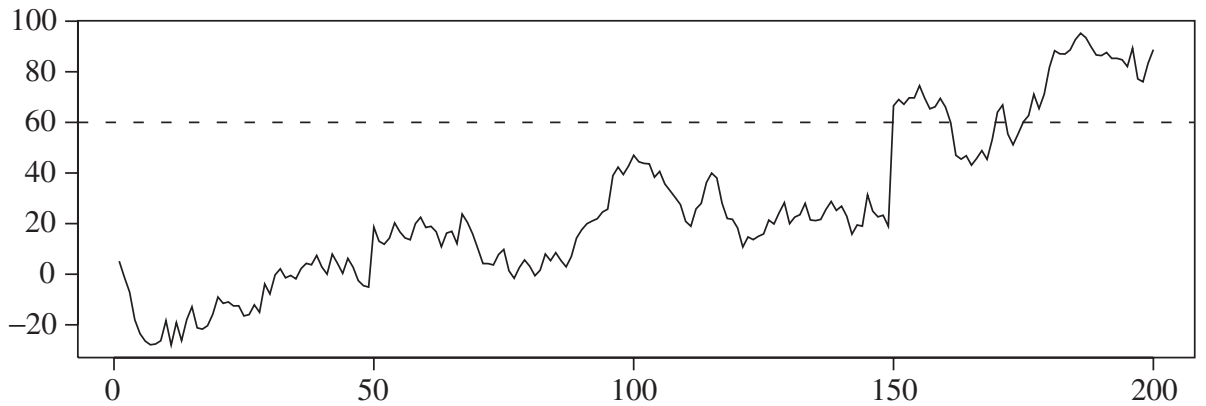

Figure 1: A typical path of $\tilde{X}$.

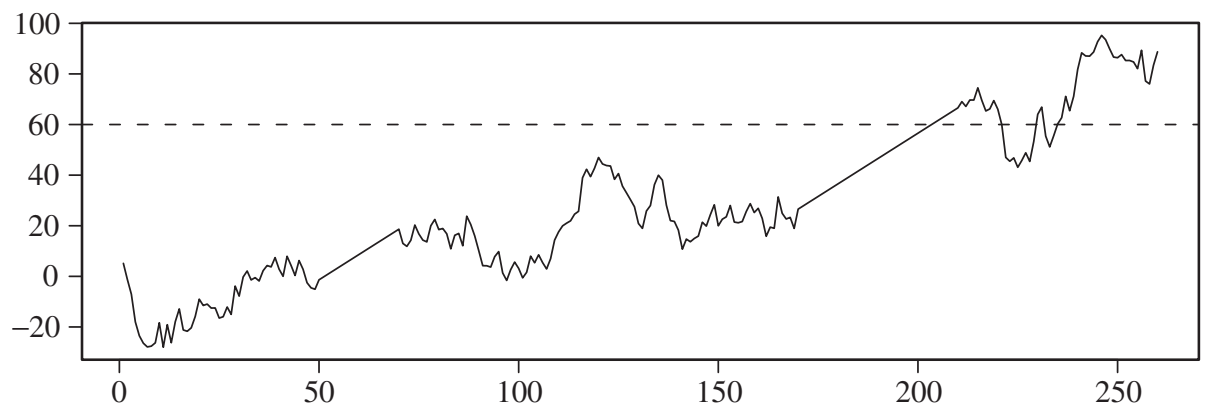

Figure 2: A typical path of $\mathcal{X}$.

We shall exclude the case where $\tilde{\mu}_{i}=\tilde{\sigma}_{i}^{2}=0$ for any phase $i \in \tilde{E}$, which would govern the zero process or a pure Lévy measure. This avoids the awkward case of a nonunique time $G_{\tilde{\tau}(u)}$ of attaining the supremum of $\tilde{X}$ before the passage time $\tilde{\tau}(u)$.

The class of MAPs with these assumptions of phase-type jumps is dense within the class of all MAPs; see [7, Proposition 1]. The main advantage of the restriction on the jump distribution is the possibility of transforming the jumps into a succession of linear pieces of exponential duration (each with slope 1 or -1 ) and retrieving the original process via a simple time change; see [3, Section 3.4.4] or [9]. The path transformation is illustrated in Figures 1 and 2. Figure 1 shows a typical path of the original level process $\tilde{X}$ and Figure 2 shows the transformed path $\mathcal{X}$.

In more exact terms, the transformation is done in the following way. Without the jumps, the Lévy process $\tilde{X}^{(i)}$ during a phase $i \in \tilde{E}$ is either a linear drift (of positive or negative slope $\tilde{\mu}_{i} \in \mathbb{R}$ ) or a Brownian motion (with parameters $\tilde{\sigma}_{i}>0$ and $\tilde{\mu}_{i} \in \mathbb{R}$ ). Considering this MAP (without the jumps) we can partition its phase space $\tilde{E}$ into the subspaces $E_{p}$ (for positive drifts), $E_{\sigma}$ (for Brownian motions), and $E_{n}$ (for negative drifts). Then we introduce two new phase spaces,

$$
E_{ \pm}:=\left\{(i, j, k, \pm): i, j \in E_{p} \cup E_{\sigma} \cup E_{n}, 1 \leq k \leq m_{i j}^{ \pm}\right\},
$$

to model the jumps. Now define the enlarged phase space $E:=E_{+} \cup E_{p} \cup E_{\sigma} \cup E_{n} \cup E_{-}$ and let $E_{c}:=E_{p} \cup E_{\sigma} \cup E_{n}$ denote the subspace of $E$ that contains all phases under which the real-time movements are continuous. 
We define the modified MAP $(\mathcal{X}, g)$ over the phase space $E$ as follows. Set the phasedependent parameters as $\left(\mu_{i}, \sigma_{i}^{2}, v_{i}\right):=\left(\tilde{\mu}_{i}, \tilde{\sigma}_{i}, \mathbf{0}\right)$ for $i \in E_{c}$ and $\left(\mu_{h}, \sigma_{h}^{2}, v_{h}\right):=( \pm 1,0, \mathbf{0})$ for $h \in E_{ \pm}$, i.e. $h=(i, j, k, \pm)$, where $i, j \in E_{c}$ and $1 \leq k \leq m_{i j}^{ \pm}$. This leads to the cumulant functions $\psi_{h}(\alpha)= \pm \alpha$ for $h \in E_{ \pm}$and

$$
\psi_{i}(\alpha)= \begin{cases}\mu_{i} \alpha, & i \in E_{p} \cup E_{n}, \\ \frac{1}{2} \sigma_{i}^{2} \alpha^{2}+\mu_{i} \alpha, & i \in E_{\sigma}\end{cases}
$$

where the earlier exclusion of a phase $i \in \tilde{E}$ with $\tilde{\mu}_{i}=\tilde{\sigma}_{i}=0$ yields $\mu_{i}>0$ for $i \in E_{p}$ and $\mu_{i}<0$ for $i \in E_{n}$. The modified phase process $g$ is determined by its generator matrix $Q=\left(q_{i j}\right)_{i, j \in E}$. For this, the construction above yields

$$
q_{i h}= \begin{cases}\tilde{q}_{i i}-\lambda_{i}, & h=i \in E_{c}, \\ \tilde{q}_{i h}\left(1-p_{i h}^{+}-p_{i h}^{-}\right), & h \in E_{c}, h \neq i, \\ \lambda_{i}^{ \pm} \alpha_{k}^{(i i) \pm}, & h=(i, i, k, \pm), \\ \tilde{q}_{i j} p_{i j}^{ \pm} \alpha_{k}^{(i j) \pm}, & h=(i, j, k, \pm),\end{cases}
$$

for $i \in E_{c}$ as well as

$$
q_{(i, j, k, \pm),(i, j, l, \pm)}=T_{k l}^{(i j) \pm} \quad \text { and } \quad q_{(i, j, k, \pm), j}=\eta_{k}^{(i j) \pm}
$$

for $i, j \in E_{c}$ and $1 \leq k, l \leq m_{i j}^{ \pm}$. For later use, we define $q_{i}:=-q_{i i}$ for all $i \in E$.

Denote the MAP constructed in such a way by $(\mathcal{X}, \mathcal{g})$. The original level process $\tilde{X}$ is retrieved via the time change

$$
c(t):=\int_{0}^{t} 1_{\left\{J_{s} \in E_{c}\right\}} \mathrm{d} s, \quad c^{-1}(s):=\inf \{t \geq 0: c(t)>s\}, \quad \text { and } \quad \tilde{X}_{t}=X_{c^{-1}(t)},
$$

for all $t \geq 0$. The inverses of the cumulant functions $\psi_{i}$ can be given explicitly as

$$
\phi_{i}(\beta)= \begin{cases} \pm \beta, & i \in E_{ \pm}, \\ \frac{\beta}{\mu_{i}}, & i \in E_{p} \cup E_{n} \\ \frac{1}{\sigma_{i}} \sqrt{2 \beta+\frac{\mu_{i}^{2}}{\sigma_{i}^{2}}-\frac{\mu_{i}}{\sigma_{i}^{2}},} & i \in E_{\sigma} .\end{cases}
$$

We shall, however, use them only for the so-called ascending phases $i \in E_{a}:=E_{+} \cup E_{p} \cup E_{\sigma}$; cf. [10, Chapter VII].

Example 1. Regarding the sample paths in Figures 1 and 2, we find $g$ in phases $i \in E_{+}$during the intervals $[50,70]$ and $[170,210]$. In all other intervals, $g$ is in phases $i \in E_{c}$. Regarding the time change, we observe in Figure 1 a first jump of height 20 at time 50. We thus obtain $c^{-1}(50)=70$ and $\tilde{X}_{50}=X_{70}$. 


\subsection{First passage times}

Of central use in the present paper will be the recent derivation of the Laplace transforms for the first passage times of MAPs with phase-type jumps as given in [11]. We call the phases $i \in E_{d}:=E_{n} \cup E_{-}$descending. Define $\tilde{\tau}(x):=\inf \left\{t \geq 0: \tilde{X}_{t}>x\right\}$ for all $x \geq 0$ and assume that $\tilde{X}_{0}=0$. Note that this is the first passage time over the level $x$ for the original MAP $\tilde{X}$, meaning that we do not count the time spent in jump phases $i \in E_{ \pm}$. This means that $\tilde{\tau}(x)=c(\tau(x))=\int_{0}^{\tau(x)} 1_{\left\{J_{s} \in E_{c}\right\}} \mathrm{d} s$, according to (5). For $\gamma \geq 0$, denote

$$
\mathrm{E}_{i j}\left(\mathrm{e}^{-\gamma \tilde{\tau}(x)}\right):=\mathrm{E}\left(\mathrm{e}^{-\gamma \tilde{\tau}(x)} ; J_{\tau(x)}=j \mid J_{0}=i, X_{0}=0\right)
$$

for all $i, j \in E$. Let $\mathrm{E}\left(\mathrm{e}^{-\gamma \tilde{\tau}(x)}\right)$ denote the matrix with these entries, and write

$$
\mathrm{E}\left(\mathrm{e}^{-\gamma \tilde{\tau}(x)}\right)=\left(\begin{array}{ll}
\mathrm{E}_{(a, a)}\left(\mathrm{e}^{-\gamma \tilde{\tau}(x)}\right) & \mathrm{E}_{(a, d)}\left(\mathrm{e}^{-\gamma \tilde{\tau}(x)}\right) \\
\mathrm{E}_{(d, a)}\left(\mathrm{e}^{-\gamma \tilde{\tau}(x)}\right) & \mathrm{E}_{(d, d)}\left(\mathrm{e}^{-\gamma \tilde{\tau}(x)}\right)
\end{array}\right)
$$

in obvious block notation with respect to the subspaces $E_{a}=E_{+} \cup E_{p} \cup E_{\sigma}$ (ascending phases) and $E_{d}=E_{n} \cup E_{-}$(descending phases).

Since a first passage to a level above cannot occur in a descending phase, we obtain first $\mathrm{P}\left(J_{\tau(x)}=j\right)=0$ for all $j \in E_{d}$ and, thus, $\mathrm{E}_{(d, d)}\left(\mathrm{e}^{-\gamma \tilde{\tau}(x)}\right)=\mathrm{E}_{(a, d)}\left(\mathrm{e}^{-\gamma \tilde{\tau}(x)}\right)=\mathbf{0}$, where $\mathbf{0}$ denotes a zero matrix of suitable dimension. Equation (6) of [11] states that

$$
\mathrm{E}_{(d, a)}\left(\mathrm{e}^{-\gamma \tilde{\tau}(x)}\right)=A(\gamma) \mathrm{e}^{U(\gamma) x} \quad \text { and } \quad \mathrm{E}_{(a, a)}\left(\mathrm{e}^{-\gamma \tilde{\tau}(x)}\right)=\mathrm{e}^{U(\gamma) x}
$$

for some subgenerator matrix $U(\gamma)$ of dimension $\left|E_{a}\right| \times\left|E_{a}\right|$ and a subtransition matrix $A(\gamma)$ of dimension $\left|E_{d}\right| \times\left|E_{a}\right|$, where $|S|$ denotes the number of elements of a set $S$. Altogether, we can write

$$
\mathrm{E}\left(\mathrm{e}^{-\gamma \tilde{\tau}(x)}\right)=\left(\begin{array}{c}
I_{a} \\
A(\gamma)
\end{array}\right)\left(\begin{array}{ll}
\mathrm{e}^{U(\gamma) x} & \mathbf{0}
\end{array}\right)
$$

where $I_{a}$ denotes the identity matrix of dimension $\left|E_{a}\right| \times\left|E_{a}\right|$.

Write $\Delta_{q}:=\operatorname{diag}\left(q_{i}\right)_{i \in E}$, and let $P=\Delta_{q}^{-1} Q+I$ denote the transition matrix of phase changes. Note that $p_{i i}=0$ for all $i \in E$. Let $e_{i}^{\top}$ denote the $i$ th canonical row base vector, with appropriate dimension according to context. According to Theorem 3 of [11], $A(\gamma)$ and $U(\gamma)$ satisfy the following equations:

$$
\begin{aligned}
& e_{h}^{\top} U(\gamma)=\sum_{l=1}^{m_{i j}^{+}} T_{k l}^{(i j)+} e_{(i, j, l,+)}^{\top}+\eta_{k}^{(i j)+} e_{j}^{\top}\left(\begin{array}{c}
I_{a} \\
A(\gamma)
\end{array}\right) \quad \text { for } h=(i, j, k,+) \in E_{+}, \\
& e_{i}^{\top} U(\gamma)=-\phi_{i}\left(q_{i}+\gamma\right) e_{i}^{\top}+\phi_{i}\left(q_{i}\right) \sum_{j \in E} p_{i j} e_{j}^{\top}\left(\begin{array}{c}
I_{a} \\
A(\gamma)
\end{array}\right) L_{i}(-U(\gamma)) \quad \text { for } i \in E_{p} \cup E_{\sigma}, \\
& e_{i}^{\top} A(\gamma)=\sum_{j \in E, j \neq i} q_{i j} e_{j}^{\top}\left(\begin{array}{c}
I_{a} \\
A(\gamma)
\end{array}\right)\left(\left(q_{i}+\gamma\right) I-\psi_{i}(-U(\gamma))\right)^{-1} \quad \text { for } i \in E_{n}, \\
& e_{i}^{\top} A(\gamma)=\sum_{j \in E, j \neq i} q_{i j} e_{j}^{\top}\left(\begin{array}{c}
I_{a} \\
A(\gamma)
\end{array}\right)\left(q_{i} I-\psi_{i}(-U(\gamma))\right)^{-1} \quad \text { for } i \in E_{-} .
\end{aligned}
$$

For the MAP $(\mathcal{X}, \mathcal{g})$ with continuous level process, the matrix function

$$
L_{i}(-U(\gamma))=\frac{q_{i}}{\phi_{i}\left(q_{i}\right)}\left(\phi_{i}\left(q_{i}+\gamma\right) I+U(\gamma)\right)\left(\left(q_{i}+\gamma\right) I-\psi_{i}(-U(\gamma))\right)^{-1}
$$


can be simplified considerably. For $i \in E_{\sigma}$, the same arguments as in [11, Example 2], lead to

$$
L_{i}(-U(\gamma))=\phi_{i}^{*}\left(q_{i}\right)\left(\phi_{i}^{*}\left(q_{i}+\gamma\right) I-U(\gamma)\right)^{-1}
$$

with

$$
\phi_{i}^{*}(\beta)=\frac{1}{\sigma_{i}} \sqrt{2 \beta+\frac{\mu_{i}^{2}}{\sigma_{i}^{2}}}+\frac{\mu_{i}}{\sigma_{i}^{2}} .
$$

Furthermore, $L_{i}(-U(\gamma))=I$ for $i \in E_{p}$ (see Example 3 of [11]), while, according to (2), $\psi_{i}(-U(\gamma))=-\mu_{i} U(\gamma)$ for $i \in E_{n}$ and $\psi_{i}(-U(\gamma))=U(\gamma)$ for $i \in E_{-}$. Hence, the equations above involve rather simple expressions only.

Considering (6), it was shown in Theorem 2 of [11] that the matrices $A(\gamma)$ and $U(\gamma)$ can be determined by successive approximation as the limit of the sequence $\left(\left(A_{n}, U_{n}\right): n \geq 0\right)$ with initial values $A_{0}:=\mathbf{0}, U_{0}:=-\operatorname{diag}\left(\phi_{i}\left(q_{i}+\gamma\right) 1_{\left\{i \in E_{\sigma} \cup E_{p}\right\}}+\phi_{i}\left(q_{i}\right) 1_{\left\{i \in E_{+}\right\}}\right)_{i \in E_{a}}$ and the following iteration:

$$
\begin{aligned}
e_{h}^{\top} U_{n+1} & =\sum_{l=1}^{m_{i j}^{+}} T_{k l}^{(i j)+} e_{(i, j, l,+)}^{\top}+\eta_{k}^{(i j)+} e_{j}^{\top}\left(\begin{array}{c}
I_{a} \\
A_{n}
\end{array}\right) \quad \text { for } h=(i, j, k,+) \in E_{+}, \\
e_{i}^{\top} U_{n+1}= & -\frac{q_{i}+\gamma}{\mu_{i}} e_{i}^{\top}+\frac{1}{\mu_{i}} \sum_{j \in E, j \neq i} q_{i j} e_{j}^{\top}\left(\begin{array}{c}
I_{a} \\
A_{n}
\end{array}\right) \quad \text { for } i \in E_{p}, \\
e_{i}^{\top} A_{n+1}= & \sum_{j \in E, j \neq i} q_{i j} e_{j}^{\top}\left(\begin{array}{c}
I_{a} \\
A_{n}
\end{array}\right)\left(\left(q_{i}+\gamma\right) I+\mu_{i} U_{n}\right)^{-1} \quad \text { for } i \in E_{n}, \\
e_{i}^{\top} A_{n+1}= & \sum_{j \in E, j \neq i} q_{i j} e_{j}^{\top}\left(\begin{array}{c}
I_{a} \\
A_{n}
\end{array}\right)\left(q_{i} I-U_{n}\right)^{-1} \quad \text { for } i \in E_{-}, \\
e_{i}^{\top} U_{n+1}= & -\phi_{i}\left(q_{i}+\gamma\right) e_{i}^{\top}+\frac{2}{\sigma_{i}^{2}} \sum_{j \in E, j \neq i} q_{i j} e_{j}^{\top}\left(\begin{array}{c}
I_{a} \\
A_{n}
\end{array}\right)\left(\phi_{i}^{*}\left(q_{i}+\gamma\right) I-U_{n}\right)^{-1} \quad \text { for } i \in E_{\sigma} .
\end{aligned}
$$

For the last equality, the relation $\phi_{i}\left(q_{i}\right) \phi_{i}^{*}\left(q_{i}\right)=2 q_{i} / \sigma_{i}^{2}$ has been used. Note that the only difference between the iterations for $E_{n}$ and $E_{-}$is the missing $\gamma$ in the last factor for $E_{-}$, reflecting that we do not discount the time for phases $i \in E_{-}$as they are jump phases in real time.

\subsection{Time-reversed MAPs}

Denote the number of phases in $E$ by $m:=|E|$. Let $\pi=\left(\pi_{1}, \ldots, \pi_{m}\right)$ denote the stationary phase distribution, which can be computed by $\pi Q=\mathbf{0}$ and $\pi \mathbf{1}=\sum_{i=1}^{m} \pi_{i}=1$, where $\mathbf{0}$ denotes the zero row vector and $\mathbf{1}$ the column vector with all entries being 1 . Define the matrix $Q^{*}=\left(q_{i j}^{*}\right)_{i, j \in E}$ by $q_{i j}^{*}:=\pi_{j} q_{j i} / \pi_{i}$ for all $i, j \in E$ or in shorter notation $Q^{*}:=\Delta_{\pi}^{-1} Q^{\top} \Delta_{\pi}$, where $\Delta_{\pi}=\operatorname{diag}\left(\pi_{1}, \ldots, \pi_{m}\right)$ is the diagonal matrix with entry $\pi_{i}$ in its $i$ th row and ' $T$, denotes transposition of a matrix. Then the Markov process with state space $E$ and generator matrix $Q^{*}$ is a time-reversed version of the original phase process $\mathcal{g}$. We denote it by $\mathcal{g}^{*}=\left(J_{t}^{*}: t \geq 0\right)$.

Based on $\mathcal{g}^{*}$ we define a time reversal $\left(\mathcal{X}^{*}, \mathcal{g}^{*}\right)$ of the original MAP $(\mathcal{X}, \mathcal{g})$ by the rule that $\mathcal{X}^{*}$ evolves like a Lévy process with parameters $-\mu_{i}$ (drift) and $\sigma_{i}^{2}$ (variation) during intervals when the time-reversed phase $J_{t}^{*}$ equals $i \in E$. Note that the sign change of the $\mu_{i}$ leads to $E_{ \pm}^{*}=E_{\mp}, E_{p}^{*}=E_{n}, E_{n}^{*}=E_{p}$, and $E_{\sigma}^{*}=E_{\sigma}$. We denote the first passage times for $\left(\mathcal{X}^{*}, g^{*}\right)$ by $\tau^{*}(x):=\inf \left\{t \geq 0: X_{t}^{*}>x\right\}$ for any level $x \geq 0$. 
The same arguments as for Equation (3.3) of [2] yield the following relation between the occupation measure (before $\tau(0)$ ) for the MAP $(\mathcal{X}, \mathscr{g})$ and the first passage time for its time reversal $\left(X^{*}, g^{*}\right)$ :

$$
\begin{gathered}
\pi_{j} \mathrm{P}\left(X_{t}^{*} \in \mathrm{d} x, X_{t}^{*}>X_{u}^{*} \text { for all } u<t, J_{t}^{*}=i \mid X_{0}^{*}=0, J_{0}^{*}=j\right) \\
=\pi_{i} \mathrm{P}\left(X_{t} \in-\mathrm{d} x, \tau(0)>t, J_{t}=j \mid X_{0}=0, J_{0}=i\right)
\end{gathered}
$$

Write $\mathrm{E}_{i}$ for the conditional expectation given $X_{0}=0$ and $J_{0}=i$. Multiplying by $\mathrm{e}^{-\gamma t}$ and integrating over $t$ yields

$$
\begin{aligned}
\pi_{i} \mathrm{E}_{i} \int_{0}^{\tau(0)} 1_{\left\{X_{t} \in-\mathrm{d} x, J_{t}=j\right\}} \mathrm{e}^{-\gamma t} \mathrm{~d} t & =\pi_{j} \mathrm{E}_{j} \int_{0}^{\infty} 1_{\left\{X_{t}^{*} \in \mathrm{d} x, X_{t}^{*}>X_{u}^{*} \text { for all } u<t, J_{t}^{*}=i\right\}} \mathrm{e}^{-\gamma t} \mathrm{~d} t \\
& =\pi_{j} \mathrm{E}_{j}\left(\mathrm{e}^{-\gamma \tau^{*}(x)} ; J_{t}^{*}=i\right) .
\end{aligned}
$$

A well-known result that we shall use in the next section is the following lemma which is Theorem VI.5(i) and Theorem VII.4(i) of [10] applied to Brownian motion and its time reversal. This lemma also yields an alternative explanation for (7).

Lemma 1. Let $\mathscr{B}=\left(B_{t}: t \geq 0\right)$ denote a Brownian motion with drift $\mu \in \mathbb{R}$ and variation $\sigma^{2}>0$. Assume that $B_{0}=0$. Furthermore, let $\&(q)$ denote a random variable which is independent of $\mathcal{B}$ and has an exponential distribution with parameter $q>0$. Write

$$
\bar{B}_{\mathcal{E}(q)}:=\max _{0 \leq t \leq \mathcal{E}(q)} B_{t} \quad \text { and } \quad \bar{B}_{\mathcal{E}(q)}^{*}:=\bar{B}_{\mathcal{E}(q)}-B_{\mathcal{E}(q)},
$$

as well as

$$
G_{\mathcal{E}(q)}:=\sup \left\{t<\mathcal{E}(q): B_{t}=\bar{B}_{\mathcal{E}(q)}\right\} \text { and } G_{\mathcal{E}(q)}^{*}:=\mathcal{E}(q)-G_{\mathcal{E}(q)} .
$$

Then the pairs $\left(G_{\mathcal{E}(q)}, \bar{B}_{\mathcal{E}(q)}\right)$ and $\left(G_{\mathcal{E}(q)}^{*}, \bar{B}_{\mathcal{E}(q)}^{*}\right)$ are independent with respective measures

$$
\mathrm{E}\left(\mathrm{e}^{-\gamma G_{\mathcal{E}(q)}} ; \bar{B}_{\mathcal{E}(q)} \in \mathrm{d} x\right)=\phi(q) \mathrm{e}^{-\phi(q+\gamma) x} \mathrm{~d} x
$$

and

$$
\mathrm{E}\left(\mathrm{e}^{-\gamma G_{\mathcal{E}(q)}^{*}} ; \bar{B}_{\mathcal{E}(q)}^{*} \in \mathrm{d} y\right)=\phi^{*}(q) \mathrm{e}^{-\phi^{*}(q+\gamma) y} \mathrm{~d} y
$$

for $\gamma \geq 0$, where $\phi(\beta)$ and $\phi^{*}(\beta)$ for $\beta>0$ are given in the last line of (6) and in (8), respectively.

\section{Main result}

Let $(\tilde{X}, \tilde{g})=\left(\left(\tilde{X}_{t}, \tilde{J}_{t}\right): t \geq 0\right)$ denote a MAP with phase-type jumps, and assume that $\tilde{X}_{0}=0$. Denote the phase space of $\tilde{g}$ by $\tilde{E}$ and its generator matrix by $\tilde{Q}$. Let

$$
\tilde{\tau}(u):=\inf \left\{t>0: \tilde{X}_{t}>u\right\}
$$

denote the first passage time over some level $u \geq 0$. Write

$$
\tilde{M}_{\tilde{\tau}(u)}:=\sup \left\{\tilde{X}_{t}: t<\tilde{\tau}(u)\right\}
$$

for the maximum of $\tilde{X}$ before the first passage over $u$. We necessarily have $0 \leq \tilde{M}_{\tilde{\tau}(u)} \leq u$, where $\tilde{M}_{\tilde{\tau}(u)}=0$ means that $\tilde{\mathcal{X}}$ does not exceed its initial value 0 before it jumps (from a 
negative value) over the threshold $u \geq 0$. The case $\tilde{M}_{\tilde{\tau}(u)}=u$ means that passage occurs by creeping, i.e. the threshold $u$ is not passed by a jump but continuously. We are further interested in

$$
\tilde{G}_{\tilde{\tau}(u)}:=\sup \left\{t<\tilde{\tau}(u): \tilde{X}_{t}=\tilde{M}_{\tilde{\tau}(u)}\right\},
$$

which is the time of attaining the maximum before passage over $u$ (cf. Lemma 2 regarding its uniqueness). Finally, we wish to determine the density function of the undershoot and the overshoot, defined as

$$
U_{\tilde{\tau}(u)}:=u-\tilde{X}_{\tilde{\tau}(u)-} \quad \text { and } \quad O_{\tilde{\tau}(u)}:=\tilde{X}_{\tilde{\tau}(u)}-u,
$$

respectively. Our aim is to derive a computable expression for the joint law of these five variables in terms of the measure

$$
\mathrm{E}\left(\mathrm{e}^{-\gamma \tilde{G} \tilde{\tau}(u)-\gamma^{*}\left(\tilde{\tau}(u)-\tilde{G}_{\tilde{\tau}(u)}\right)} ; \tilde{M}_{\tilde{\tau}(u)} \in \mathrm{d} z, U_{\tilde{\tau}(u)} \in \mathrm{d} x, O_{\tilde{\tau}(u)} \in \mathrm{d} y\right),
$$

where $\gamma, \gamma^{*} \geq 0$ are the arguments for the double Laplace transform, $x, y \geq 0$, and $0 \leq z \leq u$. Note that necessarily $x \geq u-z$.

The approach of analysis in this paper is the same as in [13] for Lévy processes. We divide the sample paths into three parts: the path until the supremum $\tilde{M}_{\tilde{\tau}(u)}<u$ is attained, the path from $\tilde{M}_{\tilde{\tau}(u)}$ to $\tilde{X}_{\tilde{\tau}(u)-}$, and the final jump which leads to an overshoot of the level $u$.

Remark 1. This decomposes the sample paths either at points of phase changes or at a maximum within the (exponentially distributed) length of a phase regime. Given the phase process, the parts between phase changes are independent according to (1). The standard result in Lemma 1 shows further that the parts before and after a maximum within a single phase regime are independent. Thus, we obtain conditional independence of all three parts in our path decomposition given the phase process. For more results on this, see [18].

Example 2. Looking at Figure 2, the maximum before first passage of the level $u=60$ occurs at time $t_{M}=120$. Thus, the path of $\mathcal{X}$ is decomposed into the parts over the time intervals $[0,120],[120,170]$, and $[170,210]$. Assume that the path is derived from a MAP on a phase space $E$ with two phases, one in $E_{+}$and one in $E_{\sigma}$. Then the different phase regimes cover the intervals $[0,50],[50,70],[70,170],[170,210]$, and [210, 260]. Their increments are conditionally independent given the phase process. Furthermore, within the regime $i \in E_{\sigma}$ over the interval $[70,170]$, the part [70,120] until the maximum is independent of the part $[120,170]$ after the maximum in the sense of Lemma 1 . As a consequence, the parts $[0,120]$, $[120,170]$, and $[170,210]$ are conditionally independent given the phase process.

The first part of the decomposition is simply a first passage problem. The second part can be determined by (4) via the time-reversed process. Between the three parts we need to take possible (and necessary) phase changes into account. This reasoning will be similar to [8]. A difference to [13] is that we measure the times $\tilde{G}_{\tilde{\tau}(u)}$ and $\tilde{\tau}(u)-\tilde{G}_{\tilde{\tau}(u)}$ in terms of their Laplace transforms. This enables us to provide an explicit formula with expressions that can be readily computed.

One preliminary lemma that we need for the main result concerns the time of attaining the maximum $\tilde{M}_{\tilde{\tau}(u)}$. See [26, Lemma 2] for the equivalent statement regarding Lévy processes. 
Lemma 2. Define $\tilde{G}_{\tilde{\tau}(u)}^{\prime}:=\inf \left\{t<\tilde{\tau}(u): \tilde{X}_{t}=\tilde{M}_{\tilde{\tau}(u)}\right\}$. Then $\tilde{G}_{\tilde{\tau}(u)}=\tilde{G}_{\tilde{\tau}(u)}^{\prime}$ almost surely.

Proof. Since all possible jumps are phase type and the zero process as well as compound Poisson processes are excluded under any regime, the transition probabilities between levels of local maxima of $\tilde{X}$ are absolutely continuous. Thus, the probability of attaining the same local maximum level twice is 0 .

Given the MAP $(\tilde{X}, \tilde{g})$, we construct the modified MAP $(\mathcal{X}, \mathcal{g})$ from it as described in Section 2.1. Recall that $P=\Delta_{q}^{-1} Q+I$, where $Q$ denotes the generator matrix of $\mathscr{g}$ (see (3) and (4)) and $\Delta_{q}$ is the diagonal matrix with entries $q_{i}=-q_{i i}$ for all $i \in E$. Define $p_{i j}^{(+,-)}:=\delta_{i j}$ for $i \in E_{\sigma}$ and $p_{i j}^{(+,-)}:=p_{i j}$ for $i \in E_{+} \cup E_{p}, j \in E_{n} \cup E_{-}$. Furthermore, define

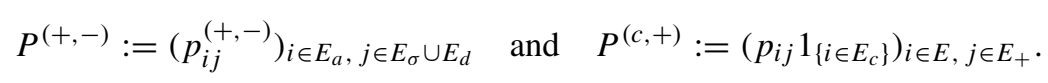

The matrices $P^{(+,-)}$and $P^{(c,+)}$ subsume the transition probabilities from ascending to descending phases and from continuous to positive jump phases, respectively.

Write $\Delta_{\phi}:=\operatorname{diag}\left(\phi_{i}\left(q_{i}\right)\right)_{i \in E_{a}}$ and $\Delta_{\phi^{*}}:=\operatorname{diag}\left(\phi_{i}\left(q_{i}\right) 1_{\left\{i \in E_{p}\right\}}+\phi_{i}^{*}\left(q_{i}\right) 1_{\left\{i \in E_{\sigma} \cup E_{n}\right\}}\right)_{i \in E}$, where $\phi_{i}^{*}\left(q_{i}\right):=q_{i} /\left(-\mu_{i}\right)$ for $i \in E_{n}$ and $\phi_{i}^{*}\left(q_{i}\right)$ is defined in (8) for $i \in E_{\sigma}$. Furthermore, define the block diagonal matrix $T=\operatorname{diag}\left(T^{(i, j)}\right)_{(i, j) \in E_{c} \times E_{c}}$ as well as the column vector $\eta=\left(\left(\eta^{(i, j)}\right)^{\top}\right)_{(i, j) \in E_{c} \times E_{c}}^{\top}$. Here $E_{c} \times E_{c}$ must be ordered in some way, say lexicographically. Note that this order must be inherited from the order on $E_{+}$. Finally, define the diagonal matrices $\Pi_{a}^{*}=\operatorname{diag}\left(1 / \pi_{i}\right)_{i \in E_{\sigma} \cup E_{d}}$ and $\Pi_{c}=\operatorname{diag}\left(\pi_{j} 1_{\left\{j \in E_{c}\right\}}\right)_{j \in E}$. Now we can state the main result.

Theorem 1. Let $\tilde{\alpha}$ denote the initial phase distribution of a $M A P(\tilde{X}, \tilde{z})$ with phase-type jumps. Define the row vector $\alpha=\left(\alpha_{i}: i \in E\right)$ on the phase space $E$ of the enlarged MAP $(\mathcal{X}, \mathcal{g})$ by $\alpha_{i}:=\tilde{\alpha}_{i}$ for all $i \in E_{c}=\tilde{E}$ and $\alpha_{i}:=0$ for $i \in E_{+} \cup E_{-}$. Then

$$
\begin{aligned}
& \mathrm{E}\left(\exp \left(-\gamma \tilde{G}_{\tilde{\tau}(u)}-\gamma^{*}\left(\tilde{\tau}(u)-\tilde{G}_{\tilde{\tau}(u)}\right)\right) ; \tilde{M}_{\tilde{\tau}(u)} \in \mathrm{d} z, U_{\tilde{\tau}(u)} \in \mathrm{d} x, O_{\tilde{\tau}(u)} \in \mathrm{d} y\right) \\
& =\alpha\left(\begin{array}{c}
I_{a} \\
A(\gamma)
\end{array}\right) \mathrm{e}^{U(\gamma) z} \Delta_{\phi} P^{(+,-)} \\
& \times\left(\Pi_{c}\left(\begin{array}{c}
A^{*}\left(\gamma^{*}\right) \\
I_{E_{\sigma} \cup E_{d}}
\end{array}\right) \mathrm{e}^{U^{*}\left(\gamma^{*}\right)(z-(u-x))} \Pi_{a}^{*}\right)^{\top} \Delta_{\phi^{*}} P^{(c,+)} \mathrm{e}^{T(x+y)} \eta \mathrm{d} x \mathrm{~d} y \mathrm{~d} z
\end{aligned}
$$

for all $\gamma, \gamma^{*} \geq 0,0<z<u, x>u-z$, and $y>0$.

Proof. We consider all possible paths leading to the event

$$
\left\{\tilde{M}_{\tilde{\tau}(u)} \in \mathrm{d} z, U_{\tilde{\tau}(u)} \in \mathrm{d} x, O_{\tilde{\tau}(u)} \in \mathrm{d} y\right\} .
$$

Since the paths of $(\tilde{X}, \tilde{\mathcal{g}})$ can be retrieved from those of $(\mathcal{X}, g)$, we may restrict our attention to $(\mathcal{X}, \mathcal{g})$. We shall, however, speak of jumps whenever we refer to linear movements governed by phases in $E_{-} \cup E_{+}$. Recall the first passage time

$$
\tau(u):=\inf \left\{t \geq 0: X_{t}>u\right\}=\min \left\{t \geq 0: X_{t}=u\right\}
$$

where the last equality holds because of the path continuity of $\mathcal{X}$. Define the times

$$
\sigma(u):=\sup \left\{t<\tau(u): J_{t} \notin E_{+}\right\} \quad \text { and } \quad \sigma^{\prime}(u):=\inf \left\{t>\tau(u): J_{t} \notin E_{+}\right\} .
$$

The assumption $z<u$ implies that $x \geq u-z>0$. Furthermore, $y>0$ almost surely since positive jumps are phase type and, hence, absolutely continuous. The time $\sigma(u)$ denotes 
the instant when the final positive jump, which leads $\mathcal{X}$ over the threshold $u$, begins. The time $\sigma^{\prime}(u)$ indicates the instant when this final jump ends and we can measure the overshoot $O_{\tilde{\tau}(u)}=\tilde{X}_{\tilde{\tau}(u)}-u=X_{\sigma^{\prime}(u)}-u$. We further obtain

$$
\tilde{M}_{\tilde{\tau}(u)}=M_{\sigma(u)}:=\sup \left\{X_{t}: t \leq \sigma(u)\right\},
$$

such that we can write

$$
\left\{\tilde{M}_{\tilde{\tau}(u)} \in \mathrm{d} z, U_{\tilde{\tau}(u)} \in \mathrm{d} x, O_{\tilde{\tau}(u)} \in \mathrm{d} y\right\}=\left\{M_{\sigma(u)} \in \mathrm{d} z, X_{\sigma(u)} \in u-\mathrm{d} x, X_{\sigma^{\prime}(u)} \in u+\mathrm{d} y\right\} .
$$

We shall employ the following path decomposition. First we consider the path up to its maximum (which is attained at a unique time, due to Lemma 2). The second part to consider is the path strictly between the time of maximum and $\sigma(u)$. The last part is the jump. Owing to Remark 1, the three parts are conditionally independent given the phase process.

The initial phase distribution is denoted by the row vector $\alpha=\left(\alpha_{i}: i \in E\right)$, where $\alpha_{i}=$ $\mathrm{P}\left(J_{0}=i\right)$ for all $i \in E$. In order to attain $M_{\sigma(u)} \in \mathrm{d} z$, a path must first pass the level $z$. This happens at the artificial time $\tau(z):=\inf \left\{t \geq 0: X_{t}>z\right\}$. The Laplace transform of the real time $\tilde{\tau}(z):=\int_{0}^{\tau(z)} 1_{\left\{J_{s} \in E_{c}\right\}} \mathrm{d} s$, restricted to $\left\{J_{\tau(z)}=i\right\}$ and with argument $\gamma \geq 0$, is given by

$$
\mathrm{E}\left(\mathrm{e}^{-\gamma \tilde{\tau}(z)} ; J_{\tau(z)}=i \mid X_{0}=0\right)=\alpha\left(\begin{array}{c}
I_{a} \\
A(\gamma)
\end{array}\right) \mathrm{e}^{U(\gamma) z} e_{i},
$$

where $e_{i}$ denotes the $i$ th canonical base column vector of dimension $\left|E_{a}\right|$.

The further development of the path depends on $J_{\tau(z)}$. There are two cases. In the first case $J_{\tau(z)}=i \in E_{+} \cup E_{p}$. Then we need an instantaneous phase change in order to satisfy $M_{\sigma(u)} \in \mathrm{d} z$. This must occur while $\mathcal{X}$ still remains in $\mathrm{d} z$. The infinitesimal rates for this to happen are $\phi_{i}\left(q_{i}\right) \mathrm{d} z=q_{i} / \mu_{i} \mathrm{~d} z$ if $i \in E_{p}$ and $\phi_{i}\left(q_{i}\right) \mathrm{d} z=q_{i} \mathrm{~d} z$ if $i \in E_{+}$; see (6). The probabilities for the next phase are then given by $p_{i j}^{(+,-)}=p_{i j}$. In the second case, $J_{\tau(z)}=i \in E_{\sigma}$, there will be no phase change immediately. In order to satisfy $M_{\sigma(u)} \in \mathrm{d} z$, we need to stop the upward ladder process of $\mathcal{X}^{(i)}$ with the infinitesimal rate $\phi_{i}\left(q_{i}\right) \mathrm{d} z$; see Lemma 1. The remaining path during phase $i$ will stay below the level $z$ and is independent of the path until $\tau(z)$. Denote the phase after this part by $J_{\tau(z)+}=i$. Then the first part of the path is described by

$$
\mathrm{E}\left(\mathrm{e}^{-\gamma \tilde{\tau}(z)} ; J_{\tau(z)+}=i \mid X_{0}=0\right)=\alpha\left(\begin{array}{c}
I_{a} \\
A(\gamma)
\end{array}\right) \mathrm{e}^{U(\gamma) z} \Delta_{\phi} P^{(+,-)} e_{i} \mathrm{~d} z .
$$

The second part of the path consists of a movement of $\mathcal{X}$ from the level $z$ at time $\tau(z)+$ to the level $u-x$ at time $\sigma(u)-$ without crossing the level $z$. Then a subsequent phase change from $E_{c}$ to $E_{+}$occurs while the level process $\mathcal{X}$ is still in $u-\mathrm{d} x$. The first event can be described via the time-reversed MAP $\left(X^{*}, g^{*}\right)$ and relation (9). Denote the matrices governing the first passage times of $\left(X^{*}, \mathscr{g}^{*}\right)$ by $A^{*}\left(\gamma^{*}\right)$ and $U^{*}\left(\gamma^{*}\right)$ for $\gamma^{*} \geq 0$. They have dimensions $\left|E_{p} \cup E_{+}\right| \times\left|E_{\sigma} \cup E_{d}\right|$ and $\left|E_{\sigma} \cup E_{d}\right|^{2}$, respectively. We obtain, from (9),

$$
\begin{aligned}
& \mathrm{E}\left(\mathrm{e}^{-\gamma^{*}(\tilde{\tau}(u)-\tilde{\tau}(z))} ; J_{\sigma(u)-}=j \mid J_{\tau(z)+}=i\right) \\
& \quad=\frac{\pi_{j}}{\pi_{i}} \mathrm{E}\left(\mathrm{e}^{-\gamma^{*} \tilde{\tau}^{*}(z-(u-x))} ; J_{\tau^{*}(z-(u-x))}^{*}=i \mid J_{0}^{*}=j\right),
\end{aligned}
$$

where $\tau^{*}(w):=\inf \left\{t \geq 0: X_{t}^{*}>w\right\}$ and $\tilde{\tau}^{*}(w)=\int_{0}^{\tau^{*}(w)} 1_{\left\{J_{s}^{*} \in E_{c}\right\}} \mathrm{d} s$. Here we consider only phases $j \in E_{c}$, since we wish the process $\mathcal{g}$ to change phase from $E_{c}$ to $E_{+}$at time $\sigma(u)$, and 
$i \in E_{\sigma} \cup E_{d}=E_{a}^{*}$ since these are the only phases in which the time-reversed process $\mathcal{X}^{*}$ can cross the level $z-(u-x)$ from below. In matrix notation, the Laplace transform of the real time spent between $\tilde{\tau}(z)$ and $\tilde{\tau}(u)$ is thus

$$
\mathrm{E}\left(\mathrm{e}^{-\gamma^{*}(\tilde{\tau}(u)-\tilde{\tau}(z))}\right)=\left(\Pi_{c}\left(\begin{array}{c}
A^{*}\left(\gamma^{*}\right) \\
I_{E_{\sigma} \cup E_{n}}
\end{array}\right) \mathrm{e}^{U^{*}\left(\gamma^{*}\right)(z-(u-x))} \Pi_{a}^{*}\right)^{\top}
$$

with $\Pi_{a}^{*}=\operatorname{diag}\left(1 / \pi_{i}\right)_{i \in E_{\sigma} \cup E_{d}}$ and $\Pi_{c}=\operatorname{diag}\left(\pi_{j} 1_{\left\{j \in E_{c}\right\}}\right)_{j \in E}$. Now to the subsequent phase change. Denote the phase at the time of moving to the level $u-x$ by $J_{\sigma(u)-}=j \in E_{c}$. In order to trigger the jump that will overshoot the level $u$ with $U_{\tilde{\tau}(u)} \in \mathrm{d} x$, the phase process $\mathcal{I}$ needs to change from $j$ to some $k \in E_{+}$while the level process $\mathcal{X}$ is still in $u-\mathrm{d} x$. This happens with probabilities $q_{j} / \mu_{j} \mathrm{~d} x p_{j k}$ for $j \in E_{p}, q_{j} /\left(-\mu_{j}\right) \mathrm{d} x p_{j k}$ for $j \in E_{n}$, and $\phi_{j}^{*}\left(q_{j}\right) \mathrm{d} x p_{j k}$ for $j \in E_{\sigma}$. Hence, the second part of the path can be subsumed in matrix notation as

$$
\begin{aligned}
& \mathrm{E}\left(\mathrm{e}^{-\gamma^{*}(\tilde{\tau}(u)-\tilde{\tau}(z))} ; J_{\sigma(u)}=k \mid J_{\tau(z)+}=i\right) \\
& \quad=e_{i}^{\top}\left(\Pi_{c}\left(\begin{array}{c}
A^{*}\left(\gamma^{*}\right) \\
I_{E_{\sigma} \cup E_{n}}
\end{array}\right) \mathrm{e}^{U^{*}\left(\gamma^{*}\right)(z-(u-x))} \Pi_{a}^{*}\right)^{\top} \Delta_{\phi^{*}} P^{(c,+)} e_{k} \mathrm{~d} x .
\end{aligned}
$$

The last part of the path is merely the final jump. Given $J_{\sigma(u)}=k \in E_{+}$, it is independent of the path before. In order to be the final jump, it must be larger than $x$. Given this, the phase-type assumption on the jumps yields the conditional density function $e_{k}^{\top} \mathrm{e}^{T(x+y)} \eta \mathrm{d} y$. This completes the proof.

If the process starts with a negative drift then the singular case, $\tilde{M}_{\tilde{\tau}(u)}=0$, is possible. This implies that $\tilde{G}_{\tilde{\tau}(u)}=0$ and $x>u$. The remaining triple law is given in the following theorem. For $\gamma^{*}=0$ and $E_{\sigma}=\varnothing$ it yields Equation (3.6) of [2] and Theorem 1 of [25].

Corollary 1. Let $\alpha$ be an initial phase distribution with support on $E_{n}$. Then

$$
\begin{aligned}
& \mathrm{E}\left(\mathrm{e}^{-\gamma^{*} \tilde{\tau}(u)} ; \tilde{M}_{\tilde{\tau}(u)}=0, U_{\tilde{\tau}(u)} \in \mathrm{d} x, O_{\tilde{\tau}(u)} \in \mathrm{d} y\right) \\
& \quad=\alpha\left(\Pi_{c}\left(\begin{array}{c}
A^{*}\left(\gamma^{*}\right) \\
I_{E_{\sigma} \cup E_{d}}
\end{array}\right) \mathrm{e}^{U^{*}\left(\gamma^{*}\right)(x-u)} \Pi_{a}^{*}\right)^{\top} \Delta_{\phi^{*}} P^{(c,+)} \mathrm{e}^{T(x+y)} \eta \mathrm{d} x \mathrm{~d} y
\end{aligned}
$$

for $x>u$ and $y>0$.

Two other singular cases that may arise are given in the following corollaries. The reasoning for them is the same as for Theorem 1 .

Corollary 2. Passage by creeping:

$$
\mathrm{E}\left(\mathrm{e}^{-\gamma \tilde{\tau}(u)} ; \tilde{M}_{\tilde{\tau}(u)}=u, U_{\tilde{\tau}(u)}=0, O_{\tilde{\tau}(u)}=0\right)=\alpha\left(\begin{array}{c}
I_{a} \\
A(\gamma)
\end{array}\right) \mathrm{e}^{U(\gamma) u} \mathbf{1}_{E_{p} \cup E_{\sigma}},
$$

where $\mathbf{1}_{E_{p} \cup E_{\sigma}}$ is a column vector of dimension $\left|E_{a}\right|$ with the ith entry being 0 for $i \in E_{+}$and 1 for $i \in E_{p} \cup E_{\sigma}$.

Corollary 3. Passage by jump from a running maximum:

$$
\begin{gathered}
\mathrm{E}\left(\mathrm{e}^{-\gamma \tilde{\tau}(u)} ; \tilde{M}_{\tilde{\tau}(u)} \in \mathrm{d} z, U_{\tilde{\tau}(u)} \in u-\mathrm{d} z, O_{\tilde{\tau}(u)} \in \mathrm{d} y\right) \\
=\alpha\left(\begin{array}{c}
I_{a} \\
A(\gamma)
\end{array}\right) \mathrm{e}^{U(\gamma) z} \Delta_{\phi} P^{(p,+)} \mathrm{e}^{T(u-z+y)} \eta \mathrm{d} y \mathrm{~d} z,
\end{gathered}
$$

where $P^{(p,+)}=\left(p_{i j} 1_{\left\{i \in E_{p}\right\}}\right)_{i \in E_{a}, j \in E_{+}}$. 


\section{Application to insurance risk}

Consider a risk reserve process with initial capital $u \geq 0$ and claims occurring like a Markovian point process (MPP); see [6], [23], and [24]. (This has traditionally been called the Markovian arrival process and abbreviated as MAP. Since we use the abbreviation MAP for the more general class of Markov additive processes, we prefer to use the term Markovian point process and the abbreviation MPP instead. Some authors use the abbreviation MArP.) It was shown in [6] that the class of MPPs is dense within the class of marked point processes. Thus, we incur no serious restriction in generality. Denote the claim arrival process by $(\mathcal{N}, \tilde{g})=\left(\left(N_{t}, \tilde{J}_{t}\right): t \geq 0\right)$ and the phase space for $\tilde{g}$ by $\tilde{E}$. Assume that the claim sizes have a phase-type distribution and denote the $n$th claim size by $C_{n}, n \in \mathbb{N}$. By [28], the class of phase-type distributions is dense within the class of all distributions on the positive real numbers. We assume further that the premium income between claims can be modelled by a Brownian motion, where the parameters $\tilde{\mu}_{i}$ (drift) and $\tilde{\sigma}_{i}$ (variation) at time $t$ may depend on the current phase $\tilde{J}_{t}=i$ of the claim arrival process. For insurance risk, we typically have $\tilde{\mu}_{i}>0$ for all $i \in \tilde{E}$. We shall allow $\tilde{\sigma}_{i}=0$ for some (or possibly all) phases, under which condition the Brownian motion becomes a linear drift. However, the case $\tilde{\mu}_{i}=\tilde{\sigma}_{i}=0$ of a constant (null) movement will be excluded. Then the process of premium income is a Markovmodulated Brownian motion which we denote by $(\mathcal{B}, \tilde{g})=\left(\left(B_{t}, \tilde{J}_{t}\right): t \geq 0\right)$. We assume that $B_{0}=0$.

Note that $\tilde{\boldsymbol{g}}$ here is the same as for the claim arrival process $(\mathcal{N}, \tilde{\mathfrak{g}})$. This is no restriction in modelling power as we can choose identical parameters $\left(\tilde{\mu}_{i}, \tilde{\sigma}_{i}\right)=\left(\tilde{\mu}_{j}, \tilde{\sigma}_{j}\right)$ for different phases $i \neq j \in \tilde{E}$ and map two different environments for the premium income and the claim arrivals by using Kronecker products. Rather on the contrary, a common phase space enables us to model correlations between claim arrivals, claim sizes, and the premium income.

With the definitions above, the risk reserve process $\mathcal{U}=\left(U_{t}: t \geq 0\right)$ is given by

$$
U_{t}=u+B_{t}-\sum_{n=1}^{N_{t}} C_{n}
$$

for $t \geq 0$. Denote the net claim process by $\tilde{X}=\left(\tilde{X}_{t}: t \geq 0\right)$, where $\tilde{X}_{t}:=u-U_{t}$ for all $t \geq 0$. Then the process $(\tilde{X}, \tilde{\mathcal{g}})$ is a MAP with phase-type jumps and we can apply the analysis presented in Section 3. For $\gamma=\gamma^{*}$, this yields in particular the Gerber-Shiu function after integrating out the variable $\tilde{M}_{\tilde{\tau}(u)}$. The following examples will illustrate this.

Example 3. We consider the classical compound Poisson model. Interclaim times and claim sizes are independent, identically exponentially distributed with parameters $\lambda>0$ and $\beta>0$, respectively. The rate of premium income is $c>0$. The net profit condition is then $\lambda /(c \beta)<1$. This model has been examined in [16]. The net claim amount at time $t \geq 0$ is given by

$$
\tilde{X}_{t}=\sum_{n=0}^{N_{t}} C_{n}-c t,
$$

where $\left(N_{t}: t \geq 0\right)$ is a Poisson process with intensity $\lambda$ and the $C_{n}, n \in \mathbb{N}$, are independent and identically distributed random variables with exponential distribution of parameter $\beta$.

The process of accumulated claims can be analysed as a MAP with exponential (and, hence, phase-type) positive jumps, where $T=-\beta$ and $\eta=\beta$. We further obtain the $\operatorname{MAP}(\mathcal{X}, \mathcal{g})$ as follows. Let the phase space be given by $E_{+}=\{1\}, E_{n}=\{2\}$, and $E_{\sigma}=\varnothing$. The parameters 
are given by $\sigma_{1}=\sigma_{2}=0, \mu_{1}=1, \mu_{2}=-c, \nu_{1}=\nu_{2}=\mathbf{0}$, and

$$
Q=\left(\begin{array}{cc}
-\beta & \beta \\
\lambda & -\lambda
\end{array}\right) \text {. }
$$

Note that phase 1 represents the upwards jumps and we shall not discount the time during sojourns in it. As shown in [11, Example 5], the Laplace transform of the first passage time $\tilde{\tau}(x):=\inf \left\{t \geq 0: \tilde{X}_{t}>x\right\}$ to a level $x>0$ is given by

$$
\mathrm{E}\left(\mathrm{e}^{-\gamma \tilde{\tau}(x)}\right)=A(\gamma) \mathrm{e}^{U(\gamma) x},
$$

where

$$
A(\gamma)=\frac{\beta-R}{\beta}, \quad U(\gamma)=-R,
$$

and

$$
-R=\frac{1}{2 c}\left(\lambda+\gamma-c \beta-\sqrt{(c \beta-\gamma-\lambda)^{2}+4 c \beta \gamma}\right),
$$

which coincides with Equation (4.24) of [16], noting that $\gamma$ is denoted as $\delta$ there. The timereversed process has a positive $\operatorname{drift}\left(E_{p}^{*}=E_{n}=\{2\}\right)$ and negative jumps $\left(E_{-}^{*}=E_{+}=\{1\}\right)$. Instead of evaluating the rather complicated expression (10), we can treat it as a spectrally negative Lévy process directly. Its cumulant function is given by

$$
\psi^{*}(x)=c x-\lambda+\lambda \frac{\beta}{\beta+x} .
$$

Hence, we find, for the inverse of $\psi^{*}$,

$$
\phi^{*}(\gamma)=\frac{1}{2 c}\left(\lambda+\gamma-c \beta+\sqrt{(c \beta-\lambda-\gamma)^{2}+4 c \beta \gamma}\right),
$$

which is denoted by $\rho$ in [16, Equation (3.12)]. We thus obtain

$$
\left(\Delta_{\pi}\left(\begin{array}{c}
A^{*}(\gamma) \\
I_{E_{n}}
\end{array}\right) \mathrm{e}^{U^{*}(\gamma)(z-(u-x))} \Delta_{\pi}^{-1}\right)^{\top}=\mathrm{e}^{-\rho(z-(u-x))} .
$$

Furthermore, we shall need $\phi_{1}\left(q_{1}\right)=\beta$ and $\phi_{2}^{*}\left(q_{2}\right)=\lambda / c$.

In order to find the function $f(x \mid u):=\mathrm{E}\left(\mathrm{e}^{-\gamma \tilde{\tau}(u)} ; U_{\tilde{\tau}(u)} \in \mathrm{d} x\right)$, we consider the cases $x \leq u$ and $x>u$ separately. We always have $\tilde{M}_{\tilde{\tau}(u)} \geq u-x$. From Theorem 1 and (12), we compute the marginal density function

$$
\begin{aligned}
\mathrm{E}\left(\mathrm{e}^{-\gamma \tilde{\tau}(u)} ; U_{\tilde{\tau}(u)} \in \mathrm{d} x, x \leq u\right) & =\int_{u-x}^{u} A(\gamma) \mathrm{e}^{U(\gamma) z} \phi_{1}\left(q_{1}\right) \mathrm{e}^{-\rho(z-u+x)} \phi_{2}^{*}\left(q_{2}\right) \mathrm{d} z \mathrm{e}^{T x} \\
& =\int_{u-x}^{u} \frac{\beta-R}{\beta} \mathrm{e}^{-R z} \beta \mathrm{e}^{-\rho(z-u+x)} \frac{\lambda}{c} \mathrm{~d} z \mathrm{e}^{-\beta x} \\
& =\frac{\lambda}{c}(\beta-R) \mathrm{e}^{-\rho(x-u)} \mathrm{e}^{-\beta x} \int_{u-x}^{u} \mathrm{e}^{-(R+\rho) z} \mathrm{~d} z \\
& =\frac{\lambda}{c} \frac{\beta-R}{R+\rho} \mathrm{e}^{-(\rho+\beta) x} \mathrm{e}^{\rho u}\left(\mathrm{e}^{-(R+\rho)(u-x)}-\mathrm{e}^{-(R+\rho) u}\right) \\
& =\frac{\lambda}{c} \frac{\beta-R}{R+\rho} \mathrm{e}^{-(\rho+\beta) x}\left(\mathrm{e}^{(R+\rho) x}-1\right) \mathrm{e}^{-R u},
\end{aligned}
$$


which coincides with Equation (6.40) of [16]. Now for the case $x>u$. This means that the event $M_{\tilde{\tau}(u)}=0$ may attain a positive probability. Corollary 1 and (12) yield, for this case,

$$
\begin{aligned}
\mathrm{E}\left(\mathrm{e}^{-\gamma \tilde{\tau}(u)}, U_{\tilde{\tau}(u)} \in \mathrm{d} x, \tilde{M}_{\tilde{\tau}(u)}=0\right) & =\mathrm{e}^{-\rho(x-u)} \phi_{2}^{*}\left(q_{2}\right) \mathrm{e}^{T x} \\
& =\mathrm{e}^{-\rho(x-u)} \frac{\lambda}{c} \mathrm{e}^{-\beta x} \\
& =\frac{\lambda}{c} \mathrm{e}^{-(\rho+\beta) x} \mathrm{e}^{\rho u} .
\end{aligned}
$$

For the case of $x>u$ and $M_{\tilde{\tau}(u)}>0$, Theorem 1 yields

$$
\begin{aligned}
\mathrm{E}\left(\mathrm{e}^{-\gamma \tilde{\tau}(u)}, U_{\tilde{\tau}(u)} \in \mathrm{d} x, \tilde{M}_{\tilde{\tau}(u)}>0, x>u\right) & =\int_{0}^{u} \frac{\beta-R}{\beta} \mathrm{e}^{-R z} \beta \mathrm{e}^{-\rho(z-u+x)} \frac{\lambda}{c} \mathrm{~d} z \mathrm{e}^{-\beta x} \\
& =\frac{\lambda}{c} \frac{\beta-R}{R+\rho} \mathrm{e}^{-(\rho+\beta) x} \mathrm{e}^{\rho u}\left(1-\mathrm{e}^{-(R+\rho) u}\right) \\
& =\frac{\lambda}{c} \frac{\beta-R}{R+\rho} \mathrm{e}^{-(\rho+\beta) x}\left(\mathrm{e}^{\rho u}-\mathrm{e}^{-R u}\right) .
\end{aligned}
$$

Adding these results we finally obtain

$$
\begin{aligned}
\mathrm{E}\left(\mathrm{e}^{-\gamma \tilde{\tau}(u)}, U_{\tilde{\tau}(u)} \in \mathrm{d} x, x>u\right) & =\frac{\lambda}{c} \mathrm{e}^{-(\rho+\beta) x}\left(\mathrm{e}^{\rho u}+\frac{\beta-R}{R+\rho}\left(\mathrm{e}^{\rho u}-\mathrm{e}^{-R u}\right)\right) \\
& =\frac{\lambda}{c(R+\rho)} \mathrm{e}^{-(\rho+\beta) x}\left((\beta+\rho) \mathrm{e}^{\rho u}-(\beta-R) \mathrm{e}^{-R u}\right),
\end{aligned}
$$

which coincides with Equation (6.39) of [16].

Example 4. The same net claim process as in (11) can be analysed by the approach in [13, Example 8]. There the time aspect is neglected and, thus, we set $\gamma=\gamma^{*}=0$. Then we obtain $\rho=0$ and $-R=(\lambda-c \beta) / c=\lambda / c-\beta$ by Equations (3.12) and (4.24) of [16]. This implies that $\beta-R=\lambda / c$, and Theorem 1 now yields, for $0<z<u$,

$$
\begin{aligned}
\mathrm{P}\left(\tilde{M}_{\tilde{\tau}(u)} \in \mathrm{d} z, U_{\tilde{\tau}(u)} \in \mathrm{d} x, O_{\tilde{\tau}(u)} \in \mathrm{d} y\right) & =\frac{\beta-R}{\beta} \mathrm{e}^{-R z} \beta \mathrm{e}^{-\rho(z-(u-x))} \frac{\lambda}{c} \mathrm{e}^{-\beta(x+y)} \beta \\
& =\frac{\lambda}{c} \mathrm{e}^{-(\beta-\lambda / c) z} \frac{\lambda}{c} \mathrm{e}^{-\beta(x+y)} \beta .
\end{aligned}
$$

Corollary 1 yields further, for $x>u$,

$$
\mathrm{P}\left(\tilde{M}_{\tilde{\tau}(u)}=0, U_{\tilde{\tau}(u)} \in \mathrm{d} x, O_{\tilde{\tau}(u)} \in \mathrm{d} y\right)=\mathrm{e}^{-\rho(x-u)} \frac{\lambda}{c} \mathrm{e}^{-\beta(x+y)} \beta=\frac{\lambda}{c} \mathrm{e}^{-\beta(x+y)} \beta .
$$

The form obtained in [13, p. 101] is

$$
\mathrm{P}\left(\tilde{M}_{\tilde{\tau}(u)} \in \mathrm{d} z, U_{\tilde{\tau}(u)} \in \mathrm{d} x, O_{\tilde{\tau}(u)} \in \mathrm{d} y\right)=\frac{1}{c} \sum_{n \geq 0} v^{* n}(\mathrm{~d} z) \Pi_{X}(x+\mathrm{d} y) \mathrm{d} x
$$

for $0 \leq z \leq u, x \geq u-z$, and $y>0$, where $\Pi_{X}(\mathrm{~d} x)=\lambda \mathrm{e}^{-\beta x} \beta \mathrm{d} x$ is the Lévy measure, $v^{* 0}=\delta_{0}$ (the Dirac measure on 0 ), and $v^{* n}$ denotes the $n$-fold convolution of the measure

$$
v(\mathrm{~d} x)=\frac{1}{c} \Pi_{X}(x, \infty) \mathrm{d} x=\frac{\lambda}{c} \mathrm{e}^{-\beta x} \mathrm{~d} x .
$$

It is immediate that the results coincide for the singular case, $M_{\tilde{\tau}(u)}=0$. In order to show 
agreement for the case $M_{\tilde{\tau}(u)}>0$, it suffices to show that

$$
\sum_{n \geq 1} v^{* n}(\mathrm{~d} z)=\frac{\lambda}{c} \mathrm{e}^{-(\beta-\lambda / c) z} \mathrm{~d} z
$$

holds. Taking Laplace transforms on both sides, we obtain on the left-hand side

$$
\int \mathrm{e}^{-\alpha z} \sum_{n \geq 1} v^{* n}(\mathrm{~d} z)=\sum_{n \geq 1} \int \mathrm{e}^{-\alpha z} v^{* n}(\mathrm{~d} z)=\sum_{n \geq 1}\left(L_{v}(\alpha)\right)^{n}=\frac{L_{\nu}(\alpha)}{1-L_{v}(\alpha)},
$$

where $L_{v}(\alpha)=\lambda / c(\alpha+\beta)^{-1}, \alpha>0$, is the Laplace transform of $v$. On the right-hand side we obtain

$$
\frac{\lambda}{c} \int \mathrm{e}^{-\alpha z} \mathrm{e}^{-(\beta-\lambda / c) z} \mathrm{~d} z=\frac{\lambda}{c} \frac{1}{\alpha+\beta-\lambda / c}=\frac{(\lambda / c)(1 /(\alpha+\beta))}{1-(\lambda / c)(1 /(\alpha+\beta))},
$$

which yields (13).

Example 5. The joint density function of the undershoot and overshoot has been derived in [8] for the fluid flow case from an insurance perspective. We set $\tilde{X}_{t}:=u-U_{t}$, where $u$ is the initial capital and $U_{t}$ is the risk reserve process as given in [8, p. 434]. For the resulting MAP $(\mathcal{X}, g)$ constructed from $\tilde{X}$ as in Section 2.1, we obtain $E_{p}=E_{\sigma}=E_{-}=\varnothing$ and $E_{+}=S_{2}$, $E_{n}=S_{1}$, where $S_{i}$ is the notation used in [8, p. 434]. Comparing the notation for the generator matrix of the phase process $\mathcal{g}$, we get the block partition

$$
Q=\left(\begin{array}{ll}
Q_{++} & Q_{+-} \\
Q_{-+} & Q_{--}
\end{array}\right)=\left(\begin{array}{ll}
T_{22} & T_{21} \\
T_{12} & T_{11}
\end{array}\right)=T .
$$

Write $P=\Delta_{q}^{-1} Q+I$ and $P_{+-}$, etc. for the obvious block partitions. The assumption that $c=1$ therein translates to $\mu_{i}=-1$ for all $i \in E_{n}$ in our notation. Since we do not need to determine the time variables, we can set $\gamma=\gamma^{*}=0$.

First of all we observe that $\Gamma$ in $[8$, p. 436] equals $A(0)$ by definition. Then Equation (16) of [11] yields

$$
U(0)=Q_{++}+Q_{+-} A(0)=T_{22}+T_{21} \Gamma=H,
$$

which is the notation used in [8, p. 437]. Furthermore, $\phi\left(q_{i}\right)=q_{i}$ for all $i \in E_{+}$and $P^{(+,-)}=P_{+-}$, which yields $\Delta_{\phi} P^{(+,-)}=Q_{+-}=T_{21}$. Regarding the time reversal $\left(\mathcal{X}^{*}, g^{*}\right)$ of $(\mathcal{X}, \mathcal{g})$, the phase subspaces translate as $E_{p}^{*}=E_{n}$ and $E_{-}^{*}=E_{+}$. Write

$$
Q^{*}=\Delta_{\pi}^{-1} Q^{\top} \Delta_{\pi}=\left(\begin{array}{ll}
Q_{--}^{*} & Q_{-+}^{*} \\
Q_{+-}^{*} & Q_{++}^{*}
\end{array}\right)
$$

such that $Q_{++}^{*}=\Delta_{\pi_{-}}^{-1} Q_{--}^{\top} \Delta_{\pi_{-}}$and $Q_{+-}^{*}=\Delta_{\pi_{-}}^{-1} Q_{-+}^{\top} \Delta_{\pi_{+}}$, denoting $\Delta_{\pi_{-}}=\operatorname{diag}\left(\pi_{i}\right)_{i \in E_{n}}$ and $\Delta_{\pi_{+}}=\operatorname{diag}\left(\pi_{i}\right)_{i \in E_{+}}$. Then $U^{*}(0)=Q_{++}^{*}+Q_{+-}^{*} A^{*}(0)$, where $A^{*}(0)$ has dimension $E_{+} \times E_{n}$. Since $E_{\sigma}=E_{\sigma}^{*}=\varnothing$, we obtain $A^{*}(0)=\Delta_{\pi_{+}}^{-1} A^{\top}(0) \Delta_{\pi_{-}}$. Thus,

$$
U^{*}(0)=\Delta_{\pi_{-}}^{-1}\left(Q_{--}^{\top}+Q_{-+}^{\top} A^{\top}(0)\right) \Delta_{\pi_{-}}
$$

and

$$
\begin{aligned}
\left(\Delta_{\pi_{-}} \mathrm{e}^{U^{*}(0)(z-(u-x))} \Delta_{\pi_{-}}^{-1}\right)^{\top} & =\left(\mathrm{e}^{\left(Q_{--}^{\top}+Q_{-+}^{\top} A^{\top}(0)\right)(z-(u-x))}\right)^{\top} \\
& =\mathrm{e}^{\left(Q_{--}+A(0) Q_{+-}\right)(z-(u-x))} \\
& =\mathrm{e}^{\left(T_{11}+\Gamma T_{21}\right)(z-(u-x))} \\
& =\mathrm{e}^{K(z-(u-x))}
\end{aligned}
$$


in the notation of [8, p. 436]. Finally, $\phi^{*}\left(q_{i}\right)=q_{i}$ for all $E_{p}^{*}=E_{n}$ and $P^{(c,+)}=P_{-+}$such that $\Delta_{\phi^{*}} P^{(c,+)}=Q_{-+}=T_{12}$. For the jump, the notation translates as $T=Q_{++}=T_{22}$ and $\eta=t_{2}$. Hence, the density function $h(u, x, y)$ of the surplus prior to ruin (the undershoot $x$ ) and the deficit at ruin (the overshoot $y$ ) is given by

$$
h(u, x, y)=\int_{z=u-x}^{u} \mathrm{P}\left(M_{\tilde{\tau}(u)} \in \mathrm{d} z, U_{\tilde{\tau}(u)} \in \mathrm{d} x, O_{\tilde{\tau}(u)} \in \mathrm{d} y\right)
$$

for $x<u$. Theorem 1 yields

$$
\begin{aligned}
h(u, x, y)= & \alpha A(0) \int_{z=u-x}^{u} \mathrm{e}^{U(0) z} \Delta_{\phi} P^{(+,-)}\left(\Delta_{\pi_{-}} \mathrm{e}^{U^{*}\left(\gamma^{*}\right)(z-(u-x))} \Delta_{\pi_{-}}^{-1}\right)^{\top} \mathrm{d} z \\
& \times \Delta_{\phi^{*}} P^{(c,+)} \mathrm{e}^{T(x+y)} \eta \\
= & \alpha \Gamma \mathrm{e}^{H(u-x)} \int_{w=0}^{x} \mathrm{e}^{H w} T_{21} \mathrm{e}^{K w} \mathrm{~d} w T_{12} \mathrm{e}^{T_{22}(x+y)} t_{2},
\end{aligned}
$$

after the substitution $w=z-(u-x)$. This coincides with Equation (17) of [8], noting definition (19) therein. The case $x>u$ includes the singular event $\left\{\tilde{M}_{\tilde{\tau}(u)}=0\right\}$. For this, Corollary 1 and (14) yield

$$
\begin{aligned}
& \mathrm{P}\left(\tilde{M}_{\tilde{\tau}(u)}=0, U_{\tilde{\tau}(u)} \in \mathrm{d} x, O_{\tilde{\tau}(u)} \in \mathrm{d} y\right) \\
& \quad=\alpha\left(\Delta_{\pi_{-}} \mathrm{e}^{U^{*}\left(\gamma^{*}\right)(z-(u-x))} \Delta_{\pi_{-}}^{-1}\right)^{\top} \Delta_{\phi^{*}} P^{(c,+)} \mathrm{e}^{T(x+y)} \eta \\
& \quad=\alpha \mathrm{e}^{K(x-u)} T_{12} \mathrm{e}^{T_{22}(x+y)} t_{2} .
\end{aligned}
$$

The other part can be determined via Theorem 1 as

$$
\begin{aligned}
\mathrm{P}\left(\tilde{M}_{\tilde{\tau}(u)}=0, U_{\tilde{\tau}(u)} \in \mathrm{d} x, O_{\tilde{\tau}(u)} \in \mathrm{d} y\right) & =\alpha \Gamma \int_{z=0}^{u} \mathrm{e}^{H z} T_{21} \mathrm{e}^{K(z-(u-x))} \mathrm{d} z T_{12} \mathrm{e}^{T_{22}(x+y)} t_{2} \\
& =\alpha \Gamma R(u) \mathrm{e}^{K(x-u)} T_{12} \mathrm{e}^{T_{22}(x+y)} t_{2},
\end{aligned}
$$

where $R(u)=\int_{w=0}^{u} \mathrm{e}^{H w} T_{21} \mathrm{e}^{K w} \mathrm{~d} w$ is the notation used in [8, Equation (19)]. Adding (15) and (16) yields the same expression as Equation (18) of [8].

Example 6. We consider the perturbed version of the classical compound Poisson model with exponential claim sizes. The net claim process is a Lévy process with parameters $\tilde{\sigma}>0, \tilde{\mu}=-c<0$, and $\tilde{v}(\mathrm{~d} x)=1_{\{x>0\}} \lambda \beta \mathrm{e}^{-\beta x} \mathrm{~d} x$. From this we construct the $\operatorname{MAP}(\mathcal{X}, \mathcal{g})$ according to Section 2.1. This has phase space $E=\{0,1\}$ with $E_{+}=\{0\}$ and $E_{\sigma}=\{1\}$. The generator matrix of $g$ is given by

$$
Q=\left(\begin{array}{cc}
-\beta & \beta \\
\lambda & -\lambda
\end{array}\right)
$$

which implies that $q_{0}=\beta$ and $q_{1}=\lambda$. The parameters for $\mathcal{X}$ are $\sigma_{0}=0, \mu_{0}=1, \sigma_{1}=\tilde{\sigma}=: \sigma$, $\mu_{1}=-c$, and $\nu_{0}=v_{1}=\mathbf{0}$. In order to determine the matrix $U=U(\gamma)$, we note that $E_{a}=E$ such that $U$ has dimension $2 \times 2$ and there is no matrix $A(\gamma)$. We need the values of $\phi_{1}\left(q_{1}+\gamma\right)$ and $\phi_{1}^{*}\left(q_{1}+\gamma\right)$, which are given in (6) and (8) as

$$
\phi_{1}\left(q_{1}+\gamma\right)=\frac{1}{\sigma} \sqrt{2(\lambda+\gamma)+\frac{c^{2}}{\sigma^{2}}}+\frac{c}{\sigma^{2}} \text { and } \phi_{1}^{*}\left(q_{1}+\gamma\right)=\frac{1}{\sigma} \sqrt{2(\lambda+\gamma)+\frac{c^{2}}{\sigma^{2}}}-\frac{c}{\sigma^{2}} \text {. }
$$


Then the matrix $U$ is determined by the fixed point equation

$$
\begin{aligned}
& e_{0}^{\top} U=-\beta e_{0}^{\top}+\beta e_{1}^{\top}=(-\beta, \beta), \\
& e_{1}^{\top} U=-\phi_{1}\left(q_{1}+\gamma\right) e_{1}^{\top}+\frac{2}{\sigma^{2}} \lambda e_{0}^{\top}\left(\phi_{1}^{*}\left(q_{1}+\gamma\right) I-U\right)^{-1} .
\end{aligned}
$$

We further obtain

$$
\Delta_{\phi} P^{(+,-)}=\left(\begin{array}{cc}
\beta & 0 \\
0 & \phi_{1}\left(q_{1}\right)
\end{array}\right)\left(\begin{array}{l}
0 \\
1
\end{array}\right)=\left(\begin{array}{c}
0 \\
\phi_{1}\left(q_{1}\right)
\end{array}\right) .
$$

For the second part in the formula of Theorem 1, we need to determine the time-reversed MAP $\left(\mathcal{X}^{*}, g^{*}\right)$ according to Section 2.3. We obtain $E_{\sigma}^{*}=E_{\sigma}=\{1\}$ and $E_{-}^{*}=E_{+}=\{0\}$ with parameters $\sigma_{0}^{*}=0, \mu_{0}^{*}=-1, \sigma_{1}^{*}=\sigma$, and $\mu_{1}^{*}=c$ for $\mathcal{X}^{*}$. The generator matrix for $\mathrm{g}^{*}$ turns out to be $Q^{*}=\Delta_{\pi}^{-1} Q^{\top} \Delta_{\pi}=Q$, where $\pi=(\lambda /(\beta+\lambda), \beta /(\beta+\lambda))$. Thus, $q_{0}^{*}=q_{0}=\beta$ and $q_{1}^{*}=q_{1}=\lambda$. Since $E_{a}^{*}=\{1\}$ and $E_{d}^{*}=\{0\}$, the matrices $U^{*}=U^{*}\left(\gamma^{*}\right)$ and $A^{*}=A^{*}\left(\gamma^{*}\right)$ are real numbers. They are determined by

$$
U^{*}=-\phi_{1}^{*}\left(\lambda+\gamma^{*}\right)+\frac{2}{\sigma^{2}} \lambda \frac{A^{*}}{\phi_{1}\left(\lambda+\gamma^{*}\right)-U^{*}} \quad \text { and } \quad A^{*}=\frac{\beta}{\beta-U^{*}},
$$

where the duality of $\mathcal{X}$ and $\mathcal{X}^{*}$ yields $\left(\phi_{1}^{*}\right)^{*}=\phi_{1}$. With the definitions of

$$
\Pi_{c}=\left(\begin{array}{cc}
0 & 0 \\
0 & \frac{\beta}{\beta+\lambda}
\end{array}\right), \quad \Pi_{a}^{*}=\frac{\beta+\lambda}{\beta}, \quad \Delta_{\phi^{*}}=\left(\begin{array}{cc}
0 & 0 \\
0 & \phi_{1}^{*}(\lambda)
\end{array}\right), \quad P^{(c,+)}=\left(\begin{array}{l}
0 \\
1
\end{array}\right),
$$

this yields

$$
\left(\Pi_{c}\left(\begin{array}{c}
A^{*} \\
1
\end{array}\right) \mathrm{e}^{U^{*}(z-(u-x))} \Pi_{a}^{*}\right)^{\top} \Delta_{\phi^{*}} P^{(c,+)}=\mathrm{e}^{U^{*}(z-(u-x))} \phi_{1}^{*}(\lambda) .
$$

Finally, the parameters for the final jump part are $T=-\beta$ and $\eta=\beta$. For the natural choice $\alpha=e_{1}^{\top}$, the expression in Theorem 1 becomes, after the simplification $\phi_{1}(\lambda) \phi_{1}^{*}(\lambda)=2 \lambda / \sigma^{2}$,

$$
\begin{gathered}
\mathrm{E}\left(\mathrm{e}^{-\gamma \tilde{G}_{\tilde{\tau}(u)}-\gamma^{*}\left(\tilde{\tau}(u)-\tilde{G}_{\tilde{\tau}(u)}\right)} ; \tilde{M}_{\tilde{\tau}(u)} \in \mathrm{d} z, U_{\tilde{\tau}(u)} \in \mathrm{d} x, O_{\tilde{\tau}(u)} \in \mathrm{d} y\right) \\
=\left(e_{1}^{\top} \mathrm{e}^{U(\gamma) z} \mathrm{e}_{1}\right) \frac{2 \lambda}{\sigma^{2}} \mathrm{e}^{U^{*}\left(\gamma^{*}\right)(z-(u-x))} \beta \mathrm{e}^{-\beta(x+y)} \mathrm{d} x \mathrm{~d} y \mathrm{~d} z .
\end{gathered}
$$

The number $U^{*}\left(\gamma^{*}\right)$ and the matrix $U(\gamma)$ are probably best determined numerically from (17) and (18).

\section{References}

[1] Ahn, S. And BADESCU, A. L. (2007). On the analysis of the Gerber-Shiu discounted penalty function for risk processes with Markovian arrivals. Insurance Math. Econom. 41, 234-249.

[2] Asmussen, S. (1991). Ladder heights and the Markov-modulated M/G/1 queue. Stoch. Process. Appl. 37, 313326.

[3] Asmussen, S. (1995). Stationary distributions via first passage times. In Advances in Queueing, ed. J. Dshalalow, CRC Press, Boca Raton, FL, pp. 79-102.

[4] Asmussen, S. (2000). Ruin Probabilities. World Scientific, River Edge, NJ.

[5] Asmussen, S. (2003). Applied Probability and Queues, 2nd edn. Springer, New York.

[6] Asmussen, S. And Koole, G. (1993). Marked point processes as limits of Markovian arrival streams. J. Appl. Prob. 30, 365-372. 
[7] Asmussen, S., Avram, F. And Pistorius, M. (2004). Russian and American put options under exponential phase-type Lévy models. Stoch. Process. Appl. 109, 79-111.

[8] BADESCU et al. (2005). The surplus prior to ruin and the deficit at ruin for a correlated risk process. Scand. Actuarial J. 2005, 433-445.

[9] Badescu, A. et al. (2005). Risk processes analyzed as fluid queues. Scand. Actuarial J. 2005, 127-141.

[10] Bertoin, J. (1996). Lévy Processes. Cambridge University Press.

[11] Breuer, L. (2008). First passage times for Markov additive processes with positive jumps of phase type. J. Appl. Prob. 45, 779-799.

[12] ChiU, S. N. And Yin, C. C. (2003). The time of ruin, the surplus prior to ruin and the deficit at ruin for the classical risk process perturbed by diffusion. Insurance Math. Econom. 33, 59-66.

[13] Doney, R. A. and Kyprianou, A. E. (2006). Overshoots and undershoots of Lévy processes. Ann. Appl. Prob. 16, 91-106.

[14] Garrido, J. and Morales, M. (2006). On the expected discounted penalty function for Lévy risk processes. N. Amer. Actuarial J. 10, 196-218.

[15] Gerber, H. U. and LANDry, B. (1998). On the discounted penalty at ruin in a jump-diffusion and the perpetual put option. Insurance Math. Econom. 22, 263-276.

[16] Gerber, H. U. and Shiu, E. S. W. (1998). On the time value of ruin. N. Amer. Actuarial J. 2, 48-78.

[17] Gerber, H. U. and Shiu, E. S. W. (2005). The time value of ruin in a Sparre Andersen model. N. Amer. Actuarial J. 9, 49-84.

[18] Klusik, P. ANd Palmowski, Z. (2009). A note on Wiener-Hopf factorization for Markov additive processes. Preprint. Available at http://arxiv.org/abs/0906.1223v1.

[19] Kyprianou, A. E. And Palmowski, Z. (2008). Fluctuations of spectrally negative Markov additive processes. In Séminaire de Probabilités XLI (Lecture Notes Math. 1934), Springer, Berlin, pp. 121-135.

[20] LI, S. AND Garrido, J. (2005). The Gerber-Shiu function in a Sparre Andersen risk process perturbed by diffusion. Scand. Actuarial. J. 2005, 161-186.

[21] Lu, Y. AND TsaI, C. C.-L. (2007). The expected discounted penalty at ruin for a Markov-modulated risk process perturbed by diffusion. N. Amer. Actuarial J. 11, 136-149.

[22] Lü, Y. H., Wu, R. AND Xu, R. (2006). The joint distributions of some extrema for the classical risk process perturbed by diffusion. Chinese J. Eng. Math. 23, 355-360.

[23] Lucantoni, D. M. (1991). New results on the single server queue with a batch Markovian arrival process. Commun. Statist. Stoch. Models 7, 1-46.

[24] Neuts, M. F. (1979). A versatile Markovian point process. J. Appl. Prob. 16, 764-779.

[25] NG, A. C. Y. AND YANG, H. (2006). On the joint distribution of surplus before and after ruin under a Markovian regime switching model. Stoch. Process. Appl. 116, 244-266.

[26] Pecherskit, E. A. And Rogozin, B. A. (1969). On joint distributions of random variables associated with fluctuations of a process with independent increments. Theory Prob. Appl. 14, 410-423.

[27] Pistorius, M. (2006). On maxima and ladder processes for a dense class of Lévy process. J. Appl. Prob. 43, 208-220.

[28] Schassberger, R. (1973). Warteschlangen. Springer, Vienna. 